\title{
Safety Verification of RGPS Service Layer Meta-model
}

\author{
Hao Yang \\ 415360889@qq.com \\ College of New Media, Zhejiang University Of Media and Communications, China
}

Keywords: BPEL; Promela; LTL formula; safety verification

\begin{abstract}
With the increasing complexity of network software, how to ensure safety of network software function and performance becomes more and more important. Based on the characteristics of network software and framework of RGPS requirement meta-model, this paper proposes safety verification of RGPS service layer meta-model. Firstly, it uses BPEL language to describe RGPS service layer meta-model into BPEL model. And then, it uses Promela language to achieve the modeling of BPEL model. Next, it inputs LTL formula to carry on safety verification analysis of RGPS service layer meta-model. Finally, the effectiveness of this verification framework is proved by an urban traffic system example.
\end{abstract}

\section{Introduction}

With the rapid development of computer network, computer software changes toward network and service. Network software[1,2] is a kind of complex software systems which is produced under this situation. Network software deployed the network resources polymerization above the network. It provides the online service for the user which changes along with individual demand. RGPS requirement meta-model framework is a framework which is composed by layered question and cooperation question. It divides into a role layer meta-model, a goal layer meta-model, a process layer meta-model and a service layer meta-model. These four layers meta-model linked to each other. Service layer meta-model describes service information and their relationships. Service layer meta-model uses to guide the service chain management of structures and service resources.

Model checking[3-5] technology is a very important formal verification analysis technology. It was first proposed by Clarke and Emerson in 1981. It can use explicit states search or implicit fixed point computation to verify system whether satisfies some properties or realizes some functions. The visible enumeration model checking method find all reachable state sets by state search traversal. It inspects reachable state set whether has wrong states. If it has a wrong state, then the system is unsafe, otherwise the system is safe. Model checking has the advantage that it is safe to carry on the verification. The success of this method should give credit to the effective of software tool, such as Spin, SMV, CWB and so on. Petri net[6] and Promela[7] are the verification models which the modeling of present system often uses. The formalization foundation of Petri net is good. When Petri net structures reachable tree or reachable graph, along with the system scale expand can create state space explosion. Promela uses Spin tool and on-the-fly technology to avoid state space explosion and reduce state space. Promela supports to optimize verification.

This paper is organized as follows. In section 2, it introduces BPEL language to establish BPEL model of RGPS service meta-model. In section 3, it implements BPEL language to Promela language. BPEL model turns into Promela model. In section 4, it uses Spin tool to verify safety of RGPS service layer meta-model. In section 5, an urban traffic system example proves the validity of safety verification method.

\section{BPEL Model of RGPS Service Layer Meta-model}

Service layer is used to guide the service chain structure and resources polymerization. It provides implementation scheme based on user requirement service. Service is a basic element of network software. Service is divided into functional and non-functional description. On the one hand, functional description realizes Process. On the other hand, non-functional description includes static QoS Property 
and dynamic Contextual Property. Service is divided into Atomic Service and Composite Service. Atomic Service is the independent modular component. It does not need rely on other Service to carry out its functions. Atomic Service includes Message and Operation. Atomic Service is posted $\mathrm{t}$ registration and storage center by its corresponding supplier. Composite Service is composed of several Atomic Service and Composite Service combination together to complete a more complex function. Those participating Service work together according to the predefined Orchestration. Orchestration has corresponding Control Structure. Typical control structure includes Sequence, Loop, Choice, Join and Any Order. RGPS service layer meta-model is shown in Figure 1.

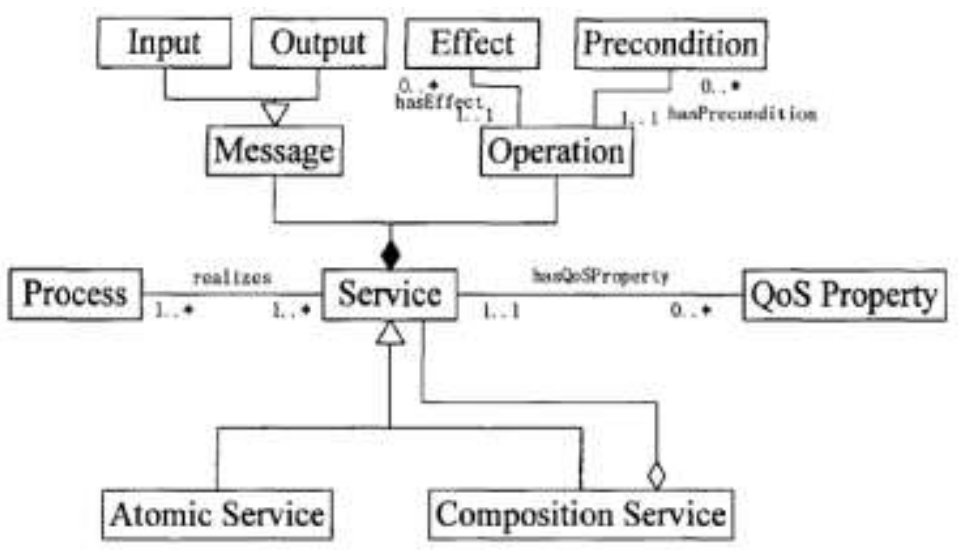

Figure 1. RGPS service layer meta-model

Based on Web service, BPEL is the process modeling language. BPEL is used to solve oral consultation uncertainty in business process plan. We use formal language to describe some business process behavior.

\section{Promela Language Modeling of BPEL Model}

Promela language is a formal language. Promela language implements modeling of finite state system. Promela language description behavior achieves processes communication and data exchange by channels. Promela model is composed of types, channels, variables and processes. Promela language types are mainly data type, process type, message type, control flow type and statement type. Processes behaviors in the BPEL model are converted processes in the Promela language.

(1) invoke

qname!inputVariable, q

q!outputVariable

(2)receive

qname?variable, $q$

(3)reply

qname!variable

(4)assign

assign!name1

assign?name2

Through JAVA language compile procedure code, it obtains Promela language modeling of BPEL model main program code.

if $($ e.getSource ()$==$ menuItembpel2)

\{

final String testBpelFile =bpelfileName;

final String testWsdlFile = wsdlfileName;

final String inputStreamFile = promelafileName;

try \{ 


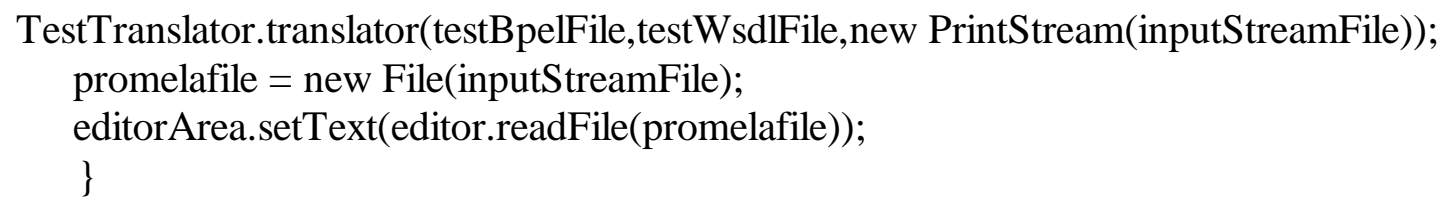

\section{Safety Verification}

After Promela language modeling, BPEL model needs input the LTL formula in the SPIN tool to carry on safety verification. Safety refers to no violation assertion, no deadlock and no bad cycle. Safety verification contains safety verification. Safety is a bad thing never happen.

We develop out RGPS service layer meta-model safety verification tool. This verification tool realizes with Java language. Firstly, it uses BPEL language to describe RGPS service layer meta-model example. Secondly, it changes BPEL language to Promela language. Thirdly, it uses LTL formula to describe model property. Finally, it takes SPIN verification and returns results.

RGPS service layer meta-model safety verification design idea is shown in Figure 2.

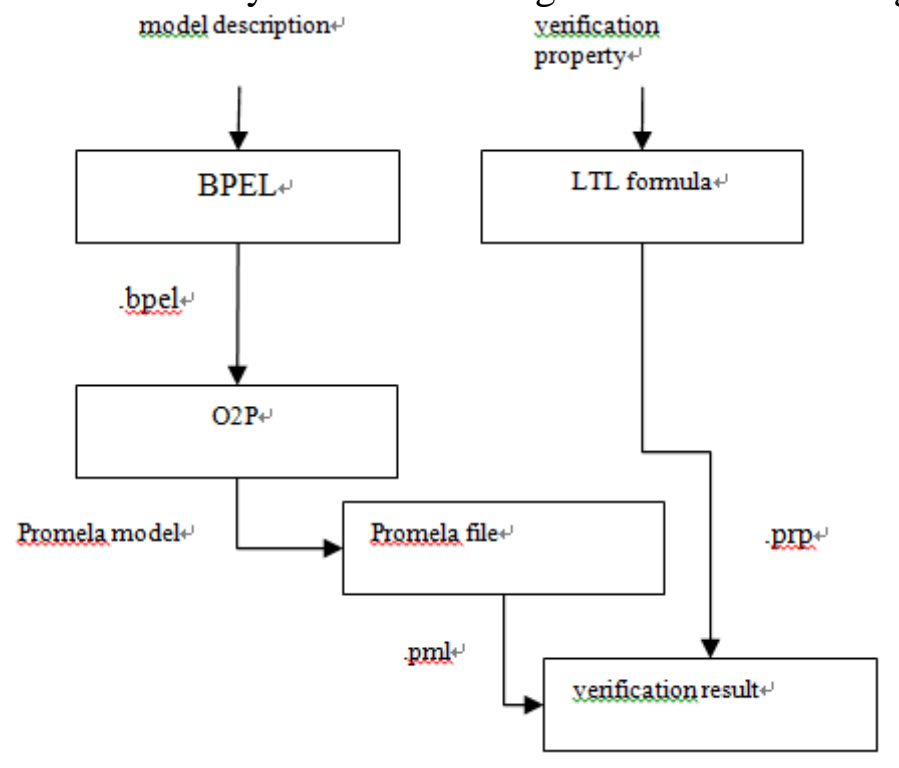

Figure 2. RGPS service layer meta-model safety verification design idea

\section{Experiments}

With the development of urban traffic system, urban traffic system network is also becoming increasingly complex. We start from urban traffic system example, use RGPS requirement meta-model framework, build urban traffic system service layer meta-model. 


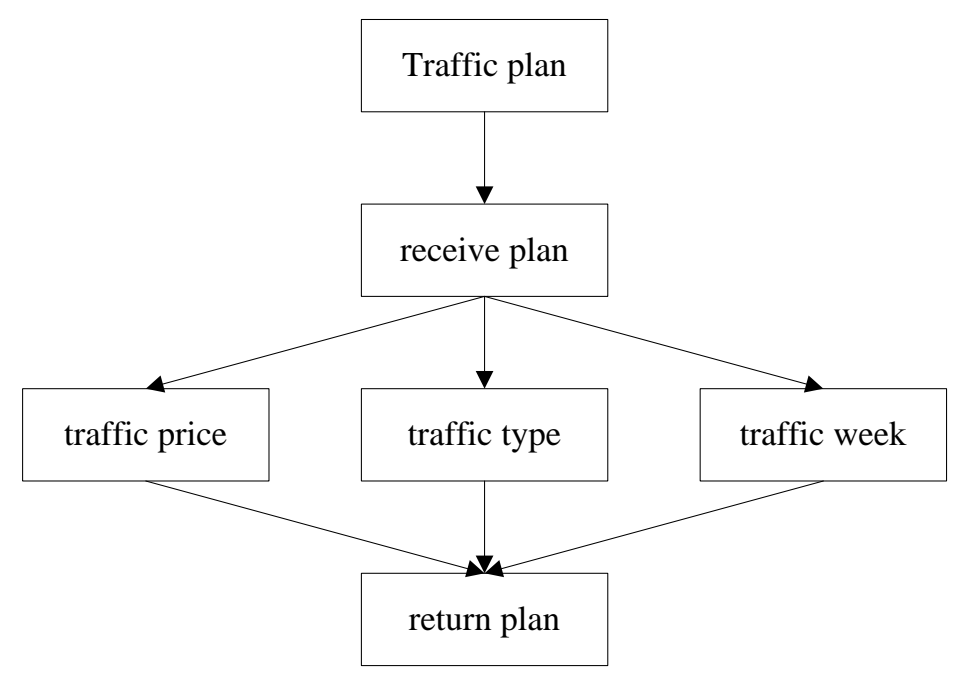

Figure 3. An urban traffic system example

In the Figure 4, after receive query action, process initialize three parallel tasks. These three tasks are computing traffic price, choosing traffic type and scheduling traffic week. Although some processes can be carried out in parallel, three tasks have interdependent control and data. When calculating the final price, it needs traffic type information. When implementing traffic plan, it needs traffic week. After complete these three tasks, it gives traffic plan to the people.

RGPS service layer meta-model partial code which is describes by BPEL is shown as follows.

<sequence>

$<$ assign $>$

$<$ copy>

$<$ from variable $=" \mathrm{TP}$ " part="TrafficMessage"/>

<to variable="TrafficRequest"

part=" TrafficMessage"/>

$</$ copy $>$

$</$ assign $>$

$<$ invoke partnerLink="Traffic"

portType $="$ tns:TrafficPT"

operation="requestTraffic"

inputVariable="TrafficRequest" $>$

outputVariable="TrafficInfo">

$<$ source linkName="TraffictoInvoice"/>

$<$ invoke >

$<$ receive partnerLink="Traffic"

portType="tns:TrafficCallbackPT"

operation="sendTraffic"

variable $="$ WeekInfo"/>

<source linkName="TraffictoWeek"/>

$</$ sequence $>$

After changing BPEL model to Promela model, it uses SPIN tool to verify safety.

Input LTL formula [](p1->p2), it obtains the following result.

Full statespace search for:

never claim -

assertion violations +(if within scope of claim)

cycle checks-(disabled by -DSAFETY)

invalid end states + (disabled by never claim)

State-vector 121 byte, depth reached 142, errors: 0

Inpute LTL formula []p, it obtains the following result. 
Full statespace search for:

never claim -

assertion violations $+($ if within scope of claim)

cycle checks -(disabled by -DSAFETY)

invalid end states -(disabled by never claim)

State-vector38 byte, depth reached 172, errors: 1

\section{Summary}

Along with Internet technology development and software production movement environment change, network software becomes software shape which based on Internet environment. Complexity of network software is too high and has state space explosion problem. Because model checking has high automation, simple rapid, provide counterexample characteristics, it is widely concerned. Complexity of model checking mainly depends on the size of system state space. The most major problem of model checking method is state space explosion and memory insufficient. Spin has provided partial order specification and on-the-fly optimization techniques. Spin is suitable for detecting increasingly complex Web services. This paper studies safety verification of RGPS service layer meta-model which based on Promela model combination and abstract analysis method. Safety verification of RGPS service layer meta-model uses technology research and tool development combination route. On the one hand, it has conducted the long term system thorough research to the core technologies. It implements BPEL language to Promela language. It unifies LTL formula properties to carry on safety verification. On the other hand, it converts breakthrough of theoretical research on practice tool development and conforms to the trend of our domestic and foreign software verification requirements.

\section{Acknowledgments}

This work is the final result of Introduction of Zhejiang University Of Media and Communications Scientific Research Grants Project(Z301B15521).

\section{References}

[1] J. Wang, K. He, B. Li,RGPS: A Unified Requirements Meta-Modeling Frame for Networked Software, Proceedings of the 3rd international workshop on Applications and advances of problem frames,(2008), pp. 29-35.

[2] K. He, R. Peng, Design methodology of Networked software evolution growth based on software patterns, Journal of System Science and Complexity,vol. 19,no.2, (2006), pp. 157-181.

[3] Meski, W. Penczek ,G. Rozenberg ,Model checking temporal properties of reaction systems, Information Sciences, vol. 313, (2015), pp. 22-42.

[4] Sistla, Employing symmetry reductions in model checking, Computer Languages Systems \& Structures, vol. 30, no.3-4, (2004), pp. 99-137.

[5] M. Mundhenk, F. Wei, An AC complete model checking problem for intuitionistic logic, Computational Complexity, vol. 23, no.4,(2014), pp. 637-669.

[6] Y. Shi, C. Tian, Z. Duan, Model checking Petri nets with MSVL, Information Sciences, vol. 363,(2016), pp. 274-291.

[7] Donaldson, A. Miller, Automatic Symmetry Detection for Promela, Journal of Automated Reasoning. vol.41, no.3-4, (2008), pp.251-293.

[8] V. Cheval, V. Cortier and S. Delaune, Deciding equivalence-based properties using constraint solving, Theoretical Computer Science,vol. 492,(2013), pp. 1-39.

[9] M. Shen, Hinfin filtering of continuous Markov jump linear system with partly known Markov modes and transition probabilities, Journal of the Franklin Institute, vol. 350, no. 10, (2013), pp. 3384-3399. 